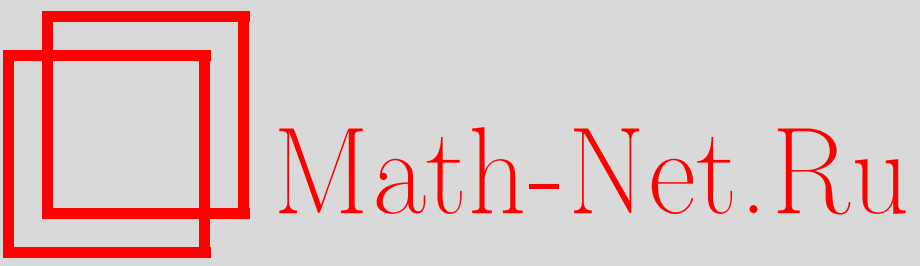

Л. А. Булавин, В. М. Сысоев, И. А. Фахретдинов, Вывод интегральных уравнений для радиальных функций распределения многокомпонентных смесей на основе масштабного преобразования фазового пространства, ТМФ, 1997, том 111, номер 3, 473-482

DOI: https://doi.org/10.4213/tmf1023

Использование Общероссийского математического портала Math-Net.Ru подразумевает, что вы прочитали и согласны с пользовательским соглашением

http://www . mathnet.ru/rus/agreement

Параметры загрузки:

IP : 35.173 .219 .149

26 апреля 2023 г., 03:25:16 
ТЕОРЕТИЧЕСКАЯ

И МАТЕМАТИЧЕСКАЯ

ФИЗИКА

Том 111, № 3

июнь, 1997

Л. А. Булавин ${ }^{*}$, В. М. Сысоев* , И. А. Фахретдинов ${ }^{\dagger}$

\section{ВЫВОД ИНТЕГРАЛЬНЫХ УРАВНЕНИЙ ДЛЯ РАДИАЛЬНЫХ ФУНКЦИЙ РАСПРЕДЕЛЕНИЯ МНОГОКОМПОНЕНТНЫХ СМЕСЕЙ НА ОСНОВЕ МАСШТАБНОГО ПРЕОБРАЗОВАНИЯ ФАЗОВОГО ПРОСТРАНСТВА}

Показано, что масштабное преобразование координатной части фазового пространства одного из компонентов смеси соответствует виртуальному изменению локальной плотности этого же компонента в термодинамической системе. Полученные результаты использованы для построения различных вариантов производящего функционала с целью вывода систем интегральных уравнений для радиальных функций распределения смесей. Получено уравнение состояния, являющееся модификацией известного уравнения Тейта. Выведены системы интегральных уравнений для радиальных функций распределения, из которых в пределе следуют известные системы уравнений Перкуса-Йевика и сверхпереплетающихся цепей.

Развитие статистической теории жидких смесей связано в основном с применением метода интегральных уравнений, связывающих потенциалы межмолекулярного взаимодействия $\varphi^{\alpha \beta}(r)$ молекул сортов $\alpha$ и $\beta$ с радиальными функциями распределения (РФР) $g^{\alpha \beta}(r)$. Метод интегральных уравнений позволяет исследовать свойства многокомпонентных систем в широкой области термодинамических параметров, включая газовые, жидкие фазовые состояния, а также окрестности критической точки [1-3].

При получении системы интегральных уравнений обычно пользуются методом Лебовитца-Перкуса [4], основанным на функциональном разложении выбранного производящего функционала $\Phi\left[F_{1}^{\alpha}\left(\mathbf{r} \mid \Psi^{\alpha}\right), \Psi^{\alpha}\right]$, зависящего от внешнего поля $\Psi^{\alpha}(\mathbf{r})$ и унарной функции распределения $F_{1}^{\alpha}\left(\mathbf{r} \mid \Psi^{\alpha}\right)$, в ряд Тейлора по степеням разности $F_{1}^{\alpha}\left(\mathbf{r} \mid \Psi^{\alpha}\right)-$ $F_{1}^{\alpha}(\mathbf{r} \mid 0)$. Надо отметить, что этот метод сталкивается с двумя сушественными трудностями. Во-первых, отсутствует физический принцип, согласно которому задавался бы конкретный вид производящего функционала. Производящий функционал обычно выбирается a priori, что требует от исследователя большой изобретательности. К удачным можно отнести выбор этих функционалов, ведущий к известным уравнениям Перкуса-Йевика (ПЙ), сверхпереплетающихся цепей (СПЦ) и разным их модификациям. Во-вторых, выбрав какой-либо производящий функционал, заранее невозможно указать критерий применимости полученных интегральных уравнений для РФР, т.к.

\footnotetext{
${ }^{*}$ Киевский национальный университет им. Т. Г. Шевченко, Киев, Украина

${ }^{\dagger}$ Башкирский государственный университет, Уфа-6, Россия
} 
$F_{1}^{\alpha}\left(\mathbf{r} \mid \Psi^{\alpha}\right)$ сложным образом выражается через $\Psi^{\alpha}$ и молекулярные функции распределения. В связи с этим для решения проблемы выбора производяшего функционала возникает необходимость поиска достаточно простых приближенных соотношений между внешним полем $\Psi^{\alpha}$ и плотностью $\rho^{\alpha}=\frac{N^{\alpha}}{V}$ компонентов смеси, где $N^{\alpha}$ - число частиц $\alpha$-компонента, $V$ - объем смеси.

Для нахождения этих соотношений воспользуемся масштабным преобразованием пространственных координат фазового пространства, соответствуюшего $\alpha$-компоненту смеси. Следуя работам $[5,6]$, можно предположить, что масштабное преобразование с масштабным фактором $\varkappa^{\alpha}(r)=\left(\frac{V^{\alpha}}{V_{0}}\right)^{1 / 3}=\left(\frac{\rho_{0}^{\alpha}}{\rho^{\alpha}}\right)^{1 / 3}$ (индекс "0" относится к начальным или базисным значениям величин объема и плотности, $V^{\alpha}$ - объем системы после масштабного преобразования координат частиц $\alpha$-компонента смеси), зависящим от пространственной переменной $r$, соответствует в некотором приближении виртуальному изменению плотности $\alpha$-компонента смеси, т.е. $\rho_{0}^{\alpha}(r) \rightarrow \rho^{\alpha}(r)$.

Для выяснения этого обстоятельства исследуем унарную функцию распределения $F_{1}^{\alpha}\left(r_{1}^{\alpha}\right) \quad \alpha$-компонента смеси в большом каноническом ансамбле при температуре $T$ и объеме $V[7]$ :

$$
\begin{aligned}
F_{1}^{\alpha}\left(r_{1}^{\alpha}\right)= & \frac{E_{1}}{E}=\frac{1}{E} \sum_{N^{1} \geq 0} \cdots \sum_{N^{\alpha} \geq 1} \cdots \sum_{N^{n} \geq 0} \frac{z_{\alpha}^{N^{\alpha}}}{\left(N^{\alpha}-1\right) !} \prod_{k \neq \alpha} \frac{z_{k}^{N^{k}}}{N^{k !}} \times \\
& \times \int D_{N} d \mathbf{r}_{2}^{\alpha} \ldots d \mathbf{r}_{N^{\alpha}}^{\alpha} \prod_{k \neq \alpha}\left\{d \mathbf{r}_{N^{k}}^{k}\right\}
\end{aligned}
$$

где $E$ - статистическая сумма:

$$
E=\sum_{N^{1} \geq 0} \cdots \sum_{N^{n} \geq 0} \prod_{k=1}^{n} \frac{z_{k}^{N^{k}}}{\left(N^{k}\right) !} \int D_{N} \prod_{k=1}^{n}\left\{d \mathbf{r}_{N^{k}}^{k}\right\}
$$

$z_{k}=\exp \left(\frac{\mu^{k}}{K_{\mathrm{b}} T}\right)-$ активность, $\mu^{k}-$ химический потенциал $k$-компонента смеси $\left(N^{1}+\cdots+N^{n}=N\right), D_{N}=D_{N}\left(\mathbf{r}_{1}, \ldots, \mathbf{r}_{N}\right) \exp \left(-\frac{1}{K_{\mathrm{L}} T} U_{N}\right), U_{N}\left(\mathbf{r}_{1}, \ldots, \mathbf{r}_{N}\right)$ - потенциальная энергия, $\left\{d \mathbf{r}_{N^{k}}^{k}\right\}=d \mathbf{r}_{1}^{k}, \ldots, d \mathbf{r}_{N^{k}}^{k}$. $K_{\text {Б }}$ - коэффициент Больцмана, $n$ - число компонентов смеси.

Произведем преобразование координат $\alpha$-компонента смеси

$$
\mathbf{r}_{i}^{\alpha} \rightarrow \varkappa^{\alpha}\left(\mathbf{r}_{i}^{\alpha}\right) \mathbf{r}_{i}^{\alpha}=\mathbf{r}_{i}^{\alpha}+k^{\alpha}\left(\mathbf{r}_{i}^{\alpha}\right) \mathbf{r}_{i}^{\alpha}
$$

и в результате получим

$$
\begin{aligned}
F_{1}^{\alpha}\left[\varkappa^{\alpha}\left(\mathbf{r}_{1}^{\alpha}\right) \mathbf{r}_{1}^{\alpha}\right]= & \frac{1}{E^{\prime}}\left\{\sum_{N^{1} \geq 0} \cdots \sum_{N^{\alpha} \geq 1} \cdots \sum_{N^{n} \geq 0} \frac{z_{\alpha}^{N^{\alpha}}}{\left(N^{\alpha}-1\right) !} \prod_{k \neq \alpha} \frac{z_{k}^{N^{k}}}{N^{k} !} \times\right. \\
& \left.\times \int D_{N}\left[\left\{r_{i}\right\},\left\{\varkappa^{\alpha}\left(\mathbf{r}_{i}^{\alpha}\right) \mathbf{r}_{i}^{\alpha}\right\}\right] \prod_{i=2}^{N^{\alpha}} d\left\{\varkappa^{\alpha}\left(\mathbf{r}_{i}^{\alpha}\right) \mathbf{r}_{i}^{\alpha}\right\} \prod_{k \neq \alpha}\left\{d \mathbf{r}_{N^{k}}^{k}\right\}\right\},
\end{aligned}
$$


здесь

$$
\begin{aligned}
E^{\prime}= & \sum_{N^{1} \geq 0} \cdots \sum_{N^{n} \geq 0} \prod_{k=1}^{n} \frac{z_{k}^{N^{k}}}{N^{k} !} \int D_{N}\left[\left\{\mathbf{r}_{i}\right\},\left\{\varkappa^{\alpha}\left(\mathbf{r}_{i}^{\alpha}\right) \mathbf{r}_{i}^{\alpha}\right\}\right] \times \\
& \times \prod_{i=1}^{N^{\alpha}} d\left\{\varkappa^{\alpha}\left(\mathbf{r}_{i}^{\alpha}\right) \mathbf{r}_{i}^{\alpha}\right\} \prod_{k \neq \alpha}\left\{d \mathbf{r}_{N^{k}}^{k}\right\},
\end{aligned}
$$

$\left\{\mathbf{r}_{i}\right\}$-набор координат всех частиц, кроме $\alpha$-компонента. Для упрошения выражения (3) разложим $D_{N}\left[\left\{\mathbf{r}_{i}\right\},\left\{\varkappa^{\alpha}\left(\mathbf{r}_{i}^{\alpha}\right) \mathbf{r}_{i}^{\alpha}\right\}\right]$ в ряд Тейлора:

$$
\begin{aligned}
& D_{N}\left[\left\{\mathbf{r}_{i}\right\},\left\{\varkappa^{\alpha}\left(\mathbf{r}_{i}^{\alpha}\right) \mathbf{r}_{i}^{\alpha}\right\}\right]=\exp \left[-\frac{1}{K_{\mathrm{5}} T} U\left(\mathbf{r}_{1}, \ldots, \mathbf{r}_{N}\right)\right]- \\
& \quad-\frac{1}{K_{\mathrm{5}} T} \exp \left(-\frac{1}{K_{\mathrm{5}} T} U\left(\mathbf{r}_{1}, \ldots, \mathbf{r}_{N}\right)\right) \sum_{i=1}^{N^{\alpha}} \nabla_{\mathbf{r}_{i}^{\alpha}} U_{N} \mathbf{r}_{i}^{\alpha} k^{\alpha}\left(\mathbf{r}_{i}^{\alpha}\right) .
\end{aligned}
$$

Тогда в приближении плавной неоднородности, пренебрегая членами, содержащими $\nabla k^{\alpha}\left(\mathbf{r}_{i}^{\alpha}\right)$, в первом порядке по $k^{\alpha}\left(\mathbf{r}_{i}^{\alpha}\right)$ мы можем формулу (3) переписать в виде

$$
F_{1}^{\alpha}\left[\varkappa^{\alpha}\left(\mathbf{r}_{1}^{\alpha}\right) \mathbf{r}_{1}^{\alpha}\right]=\frac{E_{1}^{\alpha}}{E}+a_{1}^{\alpha}-\frac{E_{1}^{\alpha}}{E} a_{2}^{\alpha}+o\left(\left[k^{\alpha}\left(\mathbf{r}_{i}^{\alpha}\right)\right]^{2}\right)
$$

где

$$
\begin{aligned}
a_{1}^{\alpha}= & 3 \int F_{2}^{\alpha \alpha}\left(\mathbf{r}_{1}^{\alpha}, \mathbf{r}_{2}^{\alpha}\right) k^{\alpha}\left(\mathbf{r}_{2}^{\alpha}\right) d \mathbf{r}_{2}^{\alpha}-\frac{1}{K_{\mathrm{5}} T E} \sum_{N^{1} \geq 0} \cdots \sum_{N^{\alpha} \geq 1} \cdots \sum_{N^{n} \geq 0} \frac{z_{\alpha}^{N^{\alpha}}}{\left(N^{\alpha}-1\right) !} \times \\
& \times \prod_{k \neq \alpha} \frac{z_{k}^{N^{k}}}{N^{k} !} \int \sum_{i=1}^{N^{\alpha}} D_{N} \nabla_{\mathbf{r}_{i}^{\alpha}} U_{N} k^{\alpha}\left(\mathbf{r}_{i}^{\alpha}\right) \mathbf{r}_{i}^{\alpha} \prod_{i=2}^{N^{\alpha}}\left\{d \mathbf{r}_{i}^{\alpha}\right\} \prod_{k \neq \alpha}\left\{d \mathbf{r}_{N^{k}}^{k}\right\} \\
a_{2}^{\alpha}= & 3 \int F_{1}^{\alpha}\left(\mathbf{r}_{2}^{\alpha}\right) k^{\alpha}\left(\mathbf{r}_{2}^{\alpha}\right) d \mathbf{r}_{2}^{\alpha}-\frac{1}{K_{\mathrm{5}} T E} \sum_{N^{1} \geq 0} \cdots \sum_{N^{n} \geq 0} \prod_{k=1}^{n} \frac{z_{k}^{N^{k}}}{N^{k !}} \times \\
& \times \int \sum_{i=1}^{N^{\alpha}} D_{N} \nabla_{\mathbf{r}_{i}^{\alpha}} U_{N} k^{\alpha}\left(\mathbf{r}_{i}^{\alpha}\right) \mathbf{r}_{i}^{\alpha} \prod_{i=1}^{N^{\alpha}\left\{d \mathbf{r}_{i}^{\alpha}\right\}} \prod_{k \neq \alpha}\left\{d \mathbf{r}_{N^{k}}^{k}\right\}
\end{aligned}
$$

$F_{2}^{\alpha \alpha}\left(\mathbf{r}_{1}^{\alpha}, \mathbf{r}_{2}^{\alpha}\right)$ - бинарная функция распределения. Используя полученное в теории фолуктуаций многокомпонентных систем [7] выражение

$$
\rho^{\alpha} \int\left[F_{2}^{\alpha \alpha}\left(\mathbf{r}_{1}^{\alpha}, \mathbf{r}_{2}^{\alpha}\right)-F_{1}^{\alpha}\left(\mathbf{r}_{1}^{\alpha}\right) F_{1}^{\alpha}\left(\mathbf{r}_{2}^{\alpha}\right)\right] d \mathbf{r}_{12}^{\alpha}=\frac{K_{\mathrm{5}} T}{\rho^{\alpha}}\left(\frac{\partial \rho^{\alpha}}{\partial \mu^{\beta}}\right)_{T, \mu^{k}, V}-1
$$

где $r_{12}^{\alpha}=\left|\mathbf{r}_{1}^{\alpha}-\mathbf{r}_{2}^{\alpha}\right|$, и теорему вириала для давления [8], на основе (5) имеем

$$
\begin{aligned}
F_{1}^{\alpha}\left[\varkappa^{\alpha}\left(\mathbf{r}_{1}^{\alpha}\right) \mathbf{r}_{1}^{\alpha}\right]= & F_{1}^{\alpha}\left(\mathbf{r}_{1}^{\alpha}\right)-3 k^{\alpha}\left(\mathbf{r}_{1}^{\alpha}\right) F_{1}^{\alpha}\left(\mathbf{r}_{1}^{\alpha}\right)\left[1-\frac{K_{\mathrm{S}} T}{\rho^{\alpha}}\left(\frac{\partial \rho^{\alpha}}{\partial \mu^{\alpha}}\right)_{T, \mu^{k}, V}\right]+ \\
& +\frac{6}{K_{\mathrm{5}} T}\left[P_{1}^{\alpha}-P^{\alpha}\right] k^{\alpha}\left(\mathbf{r}_{1}^{\alpha}\right)
\end{aligned}
$$


где $P^{\alpha}$ - парциальное давление $\alpha$-компонента смеси, $P_{1}^{\alpha}$ - парциальное давление $\alpha$-компонента во внешнем поле, создаваемое дополнительной молекулой сорта $\alpha$, закрепленной в точке $\mathbf{r}_{1}^{\alpha}$.

Анализ, проведенный в работе [9], показывает, что для систем с парноаддитивной энергией взаимодействия как для короткодействующих, так и для дальнодействующих межмолекулярных потенциалов выполняется соотношение $P^{\alpha} \approx P_{1}^{\alpha}$. Тогда соотношение (7) можно переписать в виде

$$
F_{1}^{\alpha}\left[\varkappa^{\alpha}\left(\mathbf{r}_{1}^{\alpha}\right) \mathbf{r}_{1}^{\alpha}\right]-F_{1}^{\alpha}\left(\mathbf{r}_{1}^{\alpha}\right)=-3 k^{\alpha}\left(\mathbf{r}_{1}^{\alpha}\right) F_{1}^{\alpha}\left(\mathbf{r}_{1}^{\alpha}\right)\left[1-\frac{K_{\mathrm{Б}} T}{\rho^{\alpha}}\left(\frac{\partial \rho^{\alpha}}{\partial \mu^{\alpha}}\right)_{T, \mu^{k}, V}\right] .
$$

Поскольку парциальная восприимчивость смеси $\frac{K_{\mathrm{b}} T}{\rho^{\alpha}}\left(\frac{\partial \rho^{\alpha}}{\partial \mu^{\alpha}}\right)_{T, \mu^{k}, V}$ в области плотных жидкостей и сильно сжатых газов вдали от критического состояния намного меньше единицы, выражение (8) упростится, и мы имеем

$$
F_{1}^{\alpha}\left[\varkappa^{\alpha}\left(\mathbf{r}_{1}^{\alpha}\right) \mathbf{r}_{1}^{\alpha}\right] / F_{1}^{\alpha}\left(\mathbf{r}_{1}^{\alpha}\right)-1=-3 k^{\alpha}\left(\mathbf{r}_{1}^{\alpha}\right) .
$$

Воспользовавшись приближением

$$
\left(1+3 k^{\alpha}\left(\mathbf{r}_{1}^{\alpha}\right)\right) \approx\left(1+k^{\alpha}\left(\mathbf{r}_{1}^{\alpha}\right)\right)^{3}-o\left(\left[k^{\alpha}\left(\mathbf{r}_{1}^{\alpha}\right)\right]^{2}\right) \approx\left[\varkappa^{\alpha}\left(\mathbf{r}_{1}^{\alpha}\right)\right]^{3}
$$

перепишем (9) в виде

$$
F_{1}^{\alpha}\left(\mathbf{r}_{1}^{\alpha}\right) / F_{1}^{\alpha}\left[\varkappa^{\alpha}\left(\mathbf{r}_{1}^{\alpha}\right) \mathbf{r}_{1}^{\alpha}\right]=\left[\varkappa^{\alpha}\left(\mathbf{r}_{1}^{\alpha}\right)\right]^{3}
$$

Из формулы (10) следует, что масштабное преобразование координатной части фазового пространства частиц сорта $\alpha$ с масштабным фактором $\varkappa^{\alpha}\left(\mathbf{r}_{i}^{\alpha}\right)$ в смесях с малой величиной $\frac{K_{\mathrm{Б}} T}{\rho^{\alpha}}\left(\frac{\partial \rho^{\alpha}}{\partial \mu^{\alpha}}\right)_{T, \mu^{k}, V}$ вдали от критического состояния и гранищы устойчивости (спинодали) в приближении плавной неоднородности в первом порядке $\varkappa^{\alpha}\left(\mathbf{r}_{i}^{\alpha}\right)-1$ соответствует изменению локальной плотности $\alpha$-компонента.

Используем приведенный выше вывод для выбора производяшего функционала с целью получения интегральных уравнений для РФР смесей.

Согласно [10] в методе функциональных разложений производящий функционал является аналитической функцией $u=\frac{1}{K_{\mathrm{L}} T} \Psi^{\alpha}(\mathbf{r})$ и $v=F_{1}^{\alpha}\left(\mathbf{r} \mid \Psi^{\alpha}\right)-F_{1}^{\alpha}(\mathbf{r} \mid 0)$, т.е.

$$
\Phi[u, v]=\sum_{i, j} a_{i j} u^{i} v^{j}, \quad a_{i j}=\text { const } .
$$

Если в (11) учесть только члены вида

$$
\Phi[u, v]=a_{00}+a_{10} u+a_{01} v
$$

и разложить производящий функционал в ряд по степеням отклонения унарной функции $F_{1}^{\alpha}\left(\mathbf{r} \mid \Psi^{\alpha}\right)$ от однородного распределения $F_{1}^{\alpha}(\mathbf{r} \mid 0)$, то получим

$$
\begin{aligned}
& \Phi\left[\frac{1}{K_{\mathrm{B}} T} \Psi^{\alpha}(\mathbf{r}), F_{1}^{\alpha}\left(\mathbf{r} \mid \Psi^{\alpha}\right)-F_{1}^{\alpha}(\mathbf{r} \mid 0)\right]=a_{00}+a_{01}\left(F_{1}^{\alpha}\left(\mathbf{r} \mid \Psi^{\alpha}\right)-F_{1}^{\alpha}(\mathbf{r} \mid 0)\right)+ \\
& \quad+\left.a_{10} \sum_{\gamma} \frac{\delta\left(\frac{1}{K_{\mathrm{B}} T} \Psi^{\alpha}(\mathbf{r})\right)}{\delta F_{1}^{\gamma}\left(\mathbf{r}^{\prime} \mid \Psi^{\gamma}\right)}\right|_{\Psi=0}\left[F_{1}^{\gamma}\left(\mathbf{r}^{\prime} \mid \Psi^{\gamma}\right)-F_{1}^{\gamma}\left(\mathbf{r}^{\prime} \mid 0\right)\right] d \mathbf{r}^{\prime} .
\end{aligned}
$$


Используя функциональное определение парной корреляционной функции (ПКФ)

$$
\frac{\delta\left(-\frac{1}{K_{\mathrm{b}} T} \Psi^{\alpha}(\mathbf{r})\right)}{\delta\left(\rho^{\gamma} F_{1}^{\gamma}\left(\mathbf{r}^{\prime} \mid \Psi^{\gamma}\right)\right)}=\delta_{\alpha \gamma} \frac{\delta\left(\mathbf{r}-\mathbf{r}^{\prime}\right)}{\rho^{\gamma} F_{1}^{\alpha}\left(\mathbf{r}^{\prime} \mid \Psi^{\alpha}\right)}-C^{\alpha \gamma}\left(\mathbf{r}, \mathbf{r}^{\prime}\right)
$$

и систему уравнений Орнштейна-Цернике

$$
\begin{aligned}
& F_{2}^{\alpha \beta}\left(\mathbf{r}, \mathbf{r}^{\prime}\right)-F_{1}^{\alpha}(\mathbf{r}) F_{1}^{\beta}\left(\mathbf{r}^{\prime}\right)=C^{\alpha \beta}\left(\mathbf{r}, \mathbf{r}^{\prime}\right) F_{1}^{\alpha}(\mathbf{r}) F_{1}^{\beta}\left(\mathbf{r}^{\prime}\right)+ \\
& \quad+F_{1}^{\alpha}(\mathbf{r}) \sum_{\gamma} \rho^{\gamma} \int C^{\alpha \gamma}\left(\mathbf{r}, \mathbf{r}^{\prime \prime}\right)\left[F_{2}^{\gamma \beta}\left(\mathbf{r}^{\prime \prime}, \mathbf{r}^{\prime}\right)-F_{1}^{\gamma}\left(\mathbf{r}^{\prime \prime}\right) F_{1}^{\beta}\left(\mathbf{r}^{\prime}\right)\right] d \mathbf{r}^{\prime \prime}
\end{aligned}
$$

из (12) получаем выражение для ПКФ однородной смеси

$$
\begin{aligned}
a_{10} C^{\alpha \beta}\left(\left|\mathbf{r}-\mathbf{r}^{\prime}\right|\right)= & a_{00}+a_{10}\left[g^{\alpha \beta}\left(\left|\mathbf{r}-\mathbf{r}^{\prime}\right|\right)-1\right]- \\
& -\Phi\left(\frac{1}{K_{\mathrm{5}} T} \varphi^{\alpha \beta}\left(\left|\mathbf{r}-\mathbf{r}^{\prime}\right|\right), g^{\alpha \beta}\left(\left|\mathbf{r}-\mathbf{r}^{\prime}\right|\right)-1\right) .
\end{aligned}
$$

При получении (15) предполагалось, что внешнее поле $\Psi^{\alpha}(\mathbf{r})$ создается дополнительной частицей сорта $\beta$, помешенной в точку с координатой $r^{\prime}$, т.е. $\Psi^{\alpha}(r)=\varphi^{\alpha \beta}\left(\mathbf{r}, \mathbf{r}^{\prime}\right)$, $F_{1}^{\alpha}\left(\mathbf{r} \mid \Psi^{\alpha}\right)=F_{2}^{\alpha \beta}\left(\mathbf{r}, \mathbf{r}^{\prime}\right) / F_{1}^{\alpha}(\mathbf{r}) F_{1}^{\beta}\left(\mathbf{r}^{\prime}\right)=g^{\alpha \beta}\left(\left|\mathbf{r}-\mathbf{r}^{\prime}\right|\right)$. Кроме того, в функциональном разложении (11) были отброшены члены двух типов: во-первых, члены, содержашие производные от $\Psi^{\alpha}(\mathbf{r})$ более высокого порядка, что означает отказ от учета многочастичных корреляций, важных вблизи критического состояния; во-вторых, опушены члены, имеющие большую величину при малых значениях сжимаемости.

Поскольку в функциональном разложении функциональные производные берутся при $\Psi(r)=0$, производяший функционал (11) сводится к виду

$$
\Phi[u, v]=v \sum_{i=0}^{\infty} a_{i 1} u^{i} .
$$

Для улучшения сходимости ряда (16) воспользуемся преобразованием Куммера [11]

$$
v \sum_{i} a_{i 1} u^{i}=A+v \sum_{i} a_{i 1}\left(u^{i}-u_{0}^{i}\right)
$$

где $A=v \sum_{i} a_{i 1} u_{0}^{i}, u_{0}$-вспомогательная функция, удовлетворяющая условию

$$
\left(u-u_{0}\right) / u_{0}=o\left(u_{0}\right) .
$$

Возникает вопрос выбора $u_{0}$. Можно было бы выбрать в качестве $u_{0}$ какой-нибудь модельный потенциал, как это делается в теории возмушений. Однако более удобным является выбор того же самого потенциала $\Psi^{\alpha}(\mathbf{r})=\varphi^{\alpha \beta}\left(\mathbf{r}, \mathbf{r}^{\prime}\right)$, но только подвергнутого масштабному преобразованию, что отвечает изменению плотности соответствующего компонента. Заметим, что условие (18) в случае плавных потенциалов выполняется при масштабном факторе $\varkappa^{\alpha}(r)$, достаточно близком к единице. При этом $v \sum_{i} a_{i 1} u_{0}^{i}$ соответствует производящему функционалу, вычисленному на той же изотерме, что и (11), 
но при другой плотности $\alpha$-компонента. В связи с тем, что $v \sum_{i} a_{i 1}\left(u^{i}-u_{0}^{i}\right)$ при выполнении условия (18) сходится быстрее, в качестве производящего функционала $\Phi$ выберем быстросходящийся хвост (17), т.е. производяший функционал будет иметь следуюший вид:

$$
\Phi\left[F_{1}^{\alpha}\left(\mathbf{r}^{\alpha} \mid \Psi^{\alpha}\right), \Psi^{\alpha}\right]=\sum_{i} \frac{1}{K_{\mathrm{\zeta}} T} a_{i 1}\left[\left(\Psi^{\alpha}\left(\varkappa^{\alpha} \mathbf{r}^{\alpha}\right)\right)^{i}-\left(\Psi^{\alpha}\left(\mathbf{r}^{\alpha}\right)\right)^{i}\right] F_{1}^{\alpha}\left(\mathbf{r}^{\alpha} \mid \Psi^{\alpha}\right) .
$$

Учитывая в (19) только линейные по $\Psi^{\alpha}\left(\varkappa^{\alpha} \mathbf{r}^{\alpha}\right)-\Psi^{\alpha}\left(\mathbf{r}^{\alpha}\right)$ члены, равные $\left[\exp \left(\left(\varkappa^{\alpha}-1\right) \mathbf{r}^{\alpha} \nabla_{\mathbf{r}^{\alpha}}\right)-1\right] \Psi^{\alpha}\left(\mathbf{r}^{\alpha}\right)$, и используя метод Лебовитца-Перкуса [4], получим систему интегральных уравнений

$$
\begin{aligned}
g^{\alpha \beta}(r) & \frac{1}{K_{\mathrm{B}} T} \varphi^{\alpha \beta}(r)+\widehat{\mathbf{M}}_{\mathbf{r}}\left(\varkappa^{\alpha}\right)\left[g^{\alpha \beta}(r)-1\right]-\sum_{\gamma} \rho^{\gamma} \int g^{\alpha \gamma}\left(\left|\mathbf{r}-\mathbf{r}^{\prime}\right|\right) \times \\
& \times \widehat{\mathbf{M}}_{\mathbf{r}-\mathbf{r}^{\prime}}\left(\varkappa^{\alpha}\right) \frac{1}{K_{\mathrm{E}} T} \varphi^{\alpha \gamma}\left(\left|\mathbf{r}-\mathbf{r}^{\prime}\right|\right)\left[g^{\gamma \beta}\left(r^{\prime}\right)-1\right] d \mathbf{r}^{\prime}=0 .
\end{aligned}
$$

Этой системе соответствуют ПКФ, удовлетворяющие дифференциальному уравнению

$$
\widehat{M}_{r}\left(\varkappa^{\alpha}\right) C^{\alpha \beta}(r)=-g^{\alpha \beta}(r) \widehat{M}_{r}\left(\varkappa^{\alpha}\right) \frac{1}{K_{\mathrm{L}} T} \varphi^{\alpha \beta}(r),
$$

где оператор $\widehat{M}_{r}\left(\varkappa^{\alpha}\right)=\exp \left\{\left(\varkappa^{\alpha}-1\right) \mathbf{r} \nabla_{\mathbf{r}}\right\}-1$.

На основании вышеизложенной теории по аналогии с функционалами, приводящими к системе уравнений ПЙ и СПЦ, построим производящие функционалы

$$
\begin{aligned}
\Phi_{1}\left[F_{1}^{\alpha}\left(\mathbf{r}^{\alpha} \mid \Psi^{\alpha}\right), \Psi^{\alpha}\right] & =F_{1}^{\alpha}\left(\mathbf{r}^{\alpha} \mid \Psi^{\alpha}\right) \exp \left\{-\frac{1}{K_{\mathrm{5}} T}\left[\Psi^{\alpha}\left(\varkappa^{\alpha} r^{\alpha}\right)-\Psi^{\alpha}\left(r^{\alpha}\right)\right]\right\} \\
\Phi_{2} & =\ln \left\{\Phi_{1}\left[F_{1}^{\alpha}\left(\mathbf{r}^{\alpha} \mid \Psi^{\alpha}\right), \Psi^{\alpha}\right]\right\} .
\end{aligned}
$$

При $\varkappa^{\alpha} \rightarrow \infty$ из (22) следует производяший функшионал в приближении ПЙ, а из (23)СПЦ. Методом функционального дифференцирования производящих функционалов (22) и (23) можно получить следуюшие системы интегральных уравнений для Р $Ф$ :

$$
\begin{aligned}
g^{\alpha \beta}(r) \exp \left\{-\frac{1}{K_{\mathrm{B}} T}\left[\varphi^{\alpha \beta}\left(\varkappa^{\alpha} r\right)-\varphi^{\alpha \beta}(r)\right]\right\}=g^{\alpha \beta}\left(\varkappa^{\alpha} r\right)- \\
-\sum_{\gamma} \rho^{\gamma} \int d \mathbf{s}\left[\exp \left\{-\frac{1}{K_{\mathrm{B}} T}\left[\varphi^{\alpha \gamma}\left(\varkappa^{\alpha} s\right)-\varphi^{\alpha \gamma}(s)\right]\right\}-1\right] g^{\alpha \gamma}(s)\left[g^{\gamma \beta}(|\mathbf{r}-\mathbf{s}|)-1\right], \\
\ln g^{\alpha \beta}(r)=g^{\alpha \beta}\left(\varkappa^{\alpha} r\right)-1+\frac{1}{K_{\mathrm{B}} T}\left[\varphi^{\alpha \beta}\left(\varkappa^{\alpha} r\right)-\varphi^{\alpha \beta}(r)\right]+\sum_{\gamma} \rho^{\gamma} \int d \mathbf{s}\left\{g^{\alpha \gamma}(s)-1-\right. \\
\left.\quad-\ln g^{\alpha \gamma}(s)+\frac{1}{K_{\mathrm{B}} T}\left[\varphi^{\alpha \gamma}\left(\varkappa^{\alpha} s\right)-\varphi^{\alpha \gamma}(s)\right]\right\}\left[g^{\gamma \beta}(|\mathbf{r}-\mathbf{s}|)-1\right] .
\end{aligned}
$$


Соответствующие ПКФ имеют вид

$$
\begin{aligned}
& C^{\alpha \beta}(r)-C^{\alpha \beta}\left(\varkappa^{\alpha} r\right)=g^{\alpha \beta}(r)\left[1-\exp \left[-\frac{1}{K_{\mathrm{5}} T}\left[\varphi^{\alpha \beta}\left(\varkappa^{\alpha} r\right)-\varphi^{\alpha \beta}(r)\right]\right]\right], \\
& C^{\alpha \beta}(r)-C^{\alpha \beta}\left(\varkappa^{\alpha} r\right)=g^{\alpha \beta}(r)-1-\ln g^{\alpha \beta}(r)+\frac{1}{K_{\mathrm{5}} T}\left[\varphi^{\alpha \beta}\left(\varkappa^{\alpha} r\right)-\varphi^{\alpha \beta}(r)\right] .
\end{aligned}
$$

В случае $\varkappa^{\alpha} \gg 1$, что соответствует малому значению плотности $\alpha$-компонента $\left(\rho^{\alpha} \rightarrow 0\right)$, для межмолекулярных потенциалов ограниченного радиуса действия $\left(\lim _{\varkappa^{\alpha} \rightarrow \infty} \varphi^{\alpha \beta}\left(\varkappa^{\alpha} r\right)=0, \lim _{\varkappa^{\alpha} \rightarrow \infty} C^{\alpha \beta}\left(\varkappa^{\alpha} r\right)=0, \lim _{\varkappa^{\alpha} \rightarrow \infty} g^{\alpha \beta}\left(\varkappa^{\alpha} r\right)=1\right)$ уравнения (24)-(27) переходят в систему интегральных уравнений ПЙ и СПЦ с соответствующими им ПКФ. При $\varkappa^{\alpha} \ll 1$, что отвечает большим значениям плотности $\alpha$-компонента в высокотемпературном приближении $\left(\varepsilon / K_{\mathrm{5}} T \ll 1\right.$, где $\varepsilon$ - константа взаимодействия), (24) и (26) приводят к системе интегральных уравнений для РФР, полученной в [12] для случая плотных жидких смесей без строгого обоснования.

Надо отметить, что при определенном выборе межмолекулярного потенциала для случая $\varkappa^{\alpha}=0$ можно получить с помощью (21) известное уравнение состояния Тейта [13] и рассмотреть возможность его улучшения.

Для вывода уравнения состояния бинарных смесей воспользуемся выражением для сжимаемости

$$
\frac{1}{K_{\mathrm{L}} T}\left(\frac{\partial P}{\partial \rho^{\alpha}}\right)_{T, \rho^{\beta}}=1-\sum_{\gamma} \rho^{\gamma} \int C^{\alpha \gamma}(r) d r
$$

где $P$ - давление. Выберем оператор $\widehat{\mathbf{M}}_{\mathbf{r}}$ в виде

$$
\widehat{\mathbf{M}}_{\mathbf{r}}=\exp \left(-\mathbf{r} \boldsymbol{\nabla}_{\mathbf{r}}\right)-1=\sum_{i=1}^{\infty}(-1)^{i} \frac{\left(\mathbf{r} \nabla_{\mathbf{r}}\right)^{i}}{i !}
$$

Рассмотрим, к каким результатам приводит ограничение лишь несколькими первыми $N$ членами в правой части формулы $(29)$.

При $N=1$ имеем $\widehat{\mathbf{M}}_{\mathbf{r}}=-\mathbf{r} \nabla_{\mathbf{r}}$, и для этого случая выражение (21) перепишется в виде

$$
\mathbf{r} \nabla C^{\alpha \beta}(r)=-g^{\alpha \beta}(r)\left(\mathbf{r} \nabla_{\mathbf{r}}\right) \frac{1}{K_{\mathrm{5}} T} \varphi^{\alpha \beta}(r) .
$$

Интегрируя (30) по всему объему, получим

$$
3 \int C^{\alpha \beta}(r) d \mathbf{r}=\frac{1}{K_{\mathrm{E}} T} \int g^{\alpha \beta}(r)\left(\mathbf{r} \nabla_{\mathbf{r}}\right) \varphi^{\alpha \beta}(r) d \mathbf{r} .
$$

Из выражений (28), (31) и теоремы вириала

$$
\frac{1}{K_{\mathrm{L}} T} P=\rho-\frac{1}{6} \sum_{\alpha} \sum_{\beta} \rho^{\alpha} \rho^{\beta} \int g^{\alpha \beta}(r) \mathbf{r} \nabla_{\mathbf{r}}\left(\frac{1}{K_{\mathrm{B}} T} \varphi^{\alpha \beta}(r)\right) d \mathbf{r},
$$

где $\rho$ - полная плотность смеси, следует, что

$$
\rho\left(\frac{\partial P}{\partial \rho}\right)_{T}=2 P-K_{\mathrm{Б}} T \rho
$$


Это и есть одна из модификаций уравнений состояния Тейта для смесей [14] - так называемые уравнения Мурнагана:

$$
\left(\frac{\partial P}{\partial \rho}\right)_{T}=\frac{P+B_{\mathrm{CM}}(T, X)}{\rho A_{\mathrm{CM}}}
$$

с параметрами $B_{\mathrm{CM}}=-\rho K_{\mathrm{L}} T / 2, A_{\mathrm{CM}}=1 / 2$. Полученное значение $A_{\mathrm{CM}}$ намного превьшает его экспериментальное значение для жидких смесей $A_{\mathrm{CM}} \approx \frac{1}{6} \div \frac{1}{11}[14]$ и вполне удовлетворяет данным анализа сильно сжатых смесей газов. Согласно [15] $B_{\mathrm{CM}}$ связан с давлением на спинодали. Полученное значение $B_{\mathrm{CM}}$ по порядку величины совпадает со значением $0,3 \rho K_{\mathrm{Б}} T$ для модели решеточного газа в приближении Брэгга-Вильямса [16].

Рассмотрим случай $N=2, \widehat{\mathbf{M}}_{\mathbf{r}}=\frac{1}{2}\left(\mathbf{r} \boldsymbol{\nabla}_{\mathbf{r}}\right)^{2}-\mathbf{r} \nabla_{\mathbf{r}}$. Тогда согласно выражению (21) имеем

$$
\begin{aligned}
& -\mathbf{r} \nabla_{\mathbf{r}} C^{\alpha \beta}(r)+\frac{1}{2}\left(\mathbf{r} \nabla_{\mathbf{r}}\right)^{2} C^{\alpha \beta}(r)=g^{\alpha \beta}(r) \mathbf{r} \nabla_{\mathbf{r}} \frac{1}{K_{\mathrm{B}} T} \varphi^{\alpha \beta}(r)- \\
& -g^{\alpha \beta}(r) \frac{\left(\mathbf{r} \nabla_{\mathbf{r}}\right)^{2}}{2} \frac{1}{K_{\mathrm{b}} T} \varphi^{\alpha \beta}(r) .
\end{aligned}
$$

Задавая межмолекулярный потенциал для $N \geq 2$ моделью "мягких" сфер $\varphi^{\alpha \beta}(r) \sim r^{-s}$ и интегрируя по объему соотношение (34), получаем

$$
\frac{12}{2+s} \int C^{\alpha \beta}(r) d \mathbf{r}=\int g^{\alpha \beta}(r) \mathbf{r} \nabla_{\mathbf{r}} \frac{1}{K_{\mathrm{B}} T} \varphi^{\alpha \beta}(r) d \mathbf{r} .
$$

Используя теорему вириала (32) и соотношение (35), находим опять уравнение состояния, сходное с уравнением Мурнагана с параметрами

$$
A_{\mathrm{CM}}=\frac{3}{s+2}, \quad B_{\mathrm{CM}}=\left(A_{\mathrm{CM}}-1\right) \rho K_{\mathrm{B}} T
$$

Как показано в [5], из термодинамической теории возмушений следует, что $A=\frac{3}{s}$, $B=-\rho K_{\mathrm{Б}} T$. Эти данные близки к нашим результатам, учитывая то, что для жидкостей $s \approx 30$.

Если в (11) взять произвольное $N$ число членов ряда, то для $A_{\mathrm{CM}}$ получается соотношение

$$
A_{\mathrm{CM}}^{-1}=12\left[\sum_{i=1}^{N} \frac{(s+i-2) !}{i !(s-1) !}\right] / \sum_{i=1}^{N} \frac{(i+2) !}{i !}
$$

а параметр $B_{\mathrm{CM}}$ будет по-прежнему определяться выражением (36).

Интересно отметить, что (с точки зрения подхода ББГКИ) ПК $\Phi$, определяемая (30), описывает прямую корреляцию пары частиц сортов $\alpha$ и $\beta$ через межмолекулярный потенциал и не учитывает косвенных корреляций через промежуточные частицы. Учет последуюших членов ряда (29) приводит к более последовательному учету корреляционных эффектов в ПК $\Phi C^{\alpha \beta}(r)$ и соответственно в уравнении состояния. 
В случае $s>2$ ряд (37) расходится при $N \rightarrow \infty$, т.е. $A_{\mathrm{CM}} \rightarrow 0$. Именно такой результат позволяет освободиться от главного недостатка уравнений Тейта, записанного в интегральной форме:

$$
\frac{V_{0}-V}{V_{0}}=A_{\mathrm{CM}} \ln \frac{P+B_{\mathrm{CM}}}{P_{0}+B_{\mathrm{CM}}}
$$

где $V$ - объем, $P_{0}$ и $V_{0}$ - давление и объем смеси в исходном состоянии при фиксированной температуре. Этот недостаток состоит в том, что при $P>\left(P_{0}+B_{\mathrm{CM}}\right) e^{1 / A_{\mathrm{CM}}}$, $A_{\mathrm{CM}}=$ const, уравнение (38) дает отрицательные значения объема. Этого не происходит, если параметр $A_{\mathrm{CM}}$ является убываюшей функцией плотности или давления и $A_{\mathrm{CM}}(P) \rightarrow 0$ при $P \rightarrow \infty$. Характер убывания функции $A_{\mathrm{CM}}(P)$ должен удовлетворять очевидному условию $\lim \frac{V_{0}-V}{V_{0}}=1$ при $s<\infty$. Из этих соображений при больших давлениях $A_{\mathrm{CM}}^{-1}$ следует выбрать в виде

$$
A_{\mathrm{CM}}^{-1}(P)=A_{\mathrm{CM}}^{-1}\left(P \rightarrow P_{0}\right)+\ln \frac{P+K}{P_{0}+K}+o(\ln P),
$$

где $K$ - подгоночный параметр. Из (39) следует, что $A_{\mathrm{CM}}$ при $P \ll K$ не зависит от давления или плотности, при $P \gg K$ начинает проявляться зависимость параметра $A_{\mathrm{CM}}$ от давления. Поскольку согласно [17] $A_{\mathrm{CM}}^{-1}$ связан с количеством молекул в сфере радиуса межмолекулярных сил, величина $K$ представляет собой давление, начиная с которого происходит изменение структуры ближнего порядка смеси. Окончательно изометрическое уравнение состояния жидких смесей при больших плотностях можно записать следуюшим образом:

$$
\frac{V_{0}-V}{V_{0}}=\left[\ln \frac{P+B_{\mathrm{CM}}}{P_{0}+B_{\mathrm{CM}}}\right] /\left(A_{\mathrm{CM}}^{-1}+\ln \frac{P+K}{P_{0}+K}\right) .
$$

Таким образом, на основании масштабного преобразования координатной части фазового пространства частищ $\alpha$-компонента смеси вдали от точек гранищы устойчивости найдено достаточно простое приближенное соотношение, описываюшее изменение потенциальной энергии межмолекулярного взаимодействия в зависимости от вариации локального значения плотности этого же компонента. Полученное соотношение дает возможность выбрать производяший функционал для вывода систем интегральных уравнений статистической теории смесей, справедливых при малых значениях изотермической сжимаемости. Для межмолекулярных потенциалов типа "мягких сфер" предложенная система интегральных уравнений для РФР приводит в области малой сжимаемости к уравнению состояния Мурнагана. Для смесей с большой сжимаемостью, что справедливо в газовой области, полученные системы интегральных уравнений переходят в системы интегральных уравнений ПЙ и СПЦ. 


\section{Список литературы}

[1] J. Tang, B. Lu. Mol. Phys. 1995. V. 84. P. 88.

[2] J. Anta, G. Kahl. Mol. Phys. 1995. V. 84. P. 1273.

[3] R. Chang, H. Rurstyn, J. Sengers. Phys. Rev. A. 1979. V. 19. P. 866.

[4] Р. Балеску. Равновесная и неравновесная статистическая механика. Т. I. М.: Мир, 1978.

[5] B. М. Сысоев. ТМФ. 1983. Т. 55. № 2. С. 305.

[6] Н. Н. Боголюбов. Проблемы динамической теории в статистической физике. М.-Л.: Гостехиздат, 1946.

[7] Т. Хилл. Статистическая механика. М.: Изд-во ин. лит., 1960.

[8] E. W. Grundke, D. Henderson, R. D. Murphy. Can. J. Phys. 1973. V. 51. P. 1216.

[9] Н. С. Касимов, В. М. Сисоев. ЖХХХ. 1991. Т. 65. С. 819.

[10] Н. П. Коваленко, И. З. Фишер. УФН. 1972. Т. 108. С. 209.

[11] Г. М. Фихтенгольи. Курс дифференциального и интегрального исчисления. Т. 2. М.: Наука, 1962.

[12] И. А. Фахретдинов. ЖФХ. 1997. Т. 71. С. 226.

[13] J. R. Macdonald. Rev. Mod. Phys. 1969. V. 41. P. 3316.

[14] А.З. Голик, И. И. Адаменко, В. В. Варецкий, И. И. Радченко, С. Ф. Соколовская. В кн.: "Физика жидкого состояния". Вып. 4. Киев: Изд-во Киевского ун-та, 1976. С. 91.

[15] Х.Й. Могель, А. В. Чальй. УФЖ. 1977. Т. 22. С. 100.

[16] Х.Й. Могель, А.В. Чальй, Ю.И. Шиманский. В кн.: "Физика жидкого состояния". Вып. 4. Киев: Изд-во Киевского ун-та, 1976. С. 76

[17] А.З. Голик, В. М. Сьсоев. ТВТ. 1983. Т. 21. С. 454.

Поступила в редакцию 16.XII.1996 г.

\section{A. Bulavin, V.M. Sysoev, I. A. Fakhretdinov \\ INTEGRAL EQUATIONS FOR RADIAL DISTRIBUTION FUNCTIONS OF MULTICOMPONENT MIXTURES ON THE BASIS OF PHASE SPACE SCALING TRANSFORMATION}

Scaling transformation of the phase space of a mixture component is shown to correspond to a density virtual variation of the component of a thermodynamic system. The obtained results are used to develop a technique of constructing different kinds of the generating functional to produce systems of integral equations for mixtures radial distribution functions. Empirical Tayt's equation is as well as a system of integral equations for radial distribution functions are obtained. The well-known Percus-Yevic equation and systems of equations of hypernetted chains follow from the latter equations. 